\section{A Note on the Analysis of Designed Experiments with Complex Treatment Structure}

\author{
H.P. Piepho \\ Institute of Crop Production and Grassland Research, University of Hohenheim, \\ Fruwirthstrasse 23, 70599 Stuttgart, Germany
}

E.R. Williams

Statistical Consulting Unit, The GraduateSchool, c/-MSIJohn Dedman Building, The Australian National University, Canberra ACT 0200 Australia

\author{
M. Fleck \\ Department of Organic Farming and Cropping, University of Kassel, \\ Nordbahnhofstrasse 1a, 37213 Witzenhausen, Germany
}

Additional index words. multiple comparison, linear model, linear contrasts, analysis of variance, augmented factorial

\begin{abstract}
Many experiments involve a complex treatment structure, and it is not always immediately obvious how such experiments should be analysed. This paper shows by way of three examples how a suitable linear model can be formulated that provides a meaningful analysis of variance table and allows mean comparisons of interest to be obtained in a straightforward manner. Possible advantages of this approach compared to the use of linear contrasts are discussed. It is concluded that a well-chosen model can often considerably simplify the analysis and lead to useful statistical inferences. The approach advocated in this paper is going to be strongest when there is good design structure present.
\end{abstract}

Most experimental designs considered in textbooks have a simple treatment structure, e.g., the balanced one-way design, where the treatments have no particular structure, or the two-way factorial design. In practice, however, the treatment design often differs from such standard designs, e.g., when one or several controls need to be accommodated, or the research questions to be addressed require the factorial structure to deviate from the complete case, such that not all cells in the cross-classification are filled. Three pertinent examples are as follows.

Example 1 (Marini, 2003). A randomized complete block experiment on apple (Malus $\times$ domestica Borkh.) was performed to compare three formulations of gibberellins in each of two different concentrations. In addition to this $3 \times 2$ factorial structure, the experiment comprised an untreated control. This type of treatment design is sometimes denoted as augmented factorial. The response variable was fruit set calculated as the number of fruits per limb 65 days after bloom divided by the number of flowers per cluster. One objective of the analysis is to compare the treatments to the control. In addition, the factorial structure is to be analysed. In both analyses, all data should be used to estimate experimental error.

Example 2. An experiment with maize (Zea mays $\mathrm{L}$.) was laid out as a resolvable incomplete

Received for publication 26 Oct. 2005. Accepted for publication 6 Dec. 2005. We thank Friedich Utz (University of Hohenheim, Germany) for providing the maize data. Thanks are also due to Andreas Büchse (University of Hohenheim, Germany) and to several referees for useful comments on an earlier draft. block design with two replications for 90 entries and 6 standard varieties (F. Utz, University of Hohenheim, Germany, personal communication). Incomplete blocks of size 10 were formed according to a $10 \times 10$ square lattice design, in which incomplete blocks can be grouped into complete replications comprising all treatments. Standards were replicated more often than entries, so a complete replicate contained at least two replications for some standards. The number of plots per genotype varied among standards, because a common block size was accommodated, requiring unequal sample size for the standards. It may be of interest to compare the mean yields of entries versus that of the standards. Also, a test of differences among standards and among differences of entries is useful. In addition, one may want to compute best linear unbiased predictions (BLUP) of entry effects, taking entries as random, while standards are regarded as a fixed factor.

Example 3. A completely randomized pot experiment was conducted to test the effect of various treatments on yield and quality traits of carrots (Daucus carota L.). The treatment structure comprised the following four factors, each of which had two levels (described in brackets): 1) light $(1=100 \%, 2=75 \%) ; 2)$ substrate (1 = loamy soil; 2 = sand); 3 ) horn silica ( 1 = without; 2 = with); 4) horn manure ( 1 = without, 2 = with). Horn silica and horn manure are two spray preparations used in biodynamic farming for strengthening plant vigor. The four factors were tested in a full $2^{4}$ factorial subdesign, corresponding to 16 different treatments. In addition to these treatments, carrots were fertilized with hoof-and-horn coarse meal for sufficient nitrogen supply. To explore the effect of stress, some of the treatments of the $2^{4}$ factorial were modified by omitting the nitrogen fertilization. Specifically, this was done for the four treatments exposed to $100 \%$ light and grown on loamy soil. These additional four treatments generate a $2^{3}$ factorial subdesign for nitrogen $\times$ horn silica $\times$ horn manure. One objective of the analysis is an ANOVA of the $2^{4}$ factorial subdesign (light $\times$ substrate $\times$ horn silica $\times$ horn manure). The second objective is an ANOVA of the $2^{3}$ factorial subdesign (nitrogen $\times$ horn silica $\times$ horn manure). In both analyses, all data should be used to estimate experimental error. A salient feature of this example is that the two subdesigns share four treatments.

Using the three examples described in this introduction, the present paper will show how a meaningful analysis can be obtained based on a linear model by appropriate coding of factors. Our main objective is to demonstrate that the introduction of dummy variables can conveniently solve a wide variety of inferential problems that would otherwise either require rather more effort, e.g., when multiple linear contrasts are specified (Marini, 2003), or not make fully efficient use of the data, e.g., when only data from orthogonal subdesigns are analysed. We will use a model formulation that represents nesting and crossing of factors in a simple way (McCullagh and Nelder, 1989; Piepho et al., 2003). Treatment factors may be defined as either fixed or random, and levels are either quantitative of qualitative. We will consider examples for each of these situations, thus illustrating the broad applicability or the our approach. Tests of significance in nonorthogonal linear models require special attention to be paid to the choice among Type I versus Type III hypotheses and sums-of-squares. It will be demonstrated in examples that most inferential problems can be tackled using Type I hypotheses. The ideas presented here are not novel (McCullagh and Nelder, 1989; Littell et al., 2002; Williams et al., 2002), but their application in practice seems to have been relatively limited so far. The purpose of the present paper is therefore to help disseminate these useful ideas among plant scientists.

\section{Example 1}

The original apple data were not available from the author (R. P. Marini, personal communication), so we simulated data that reproduced the same treatment means and sums-of-squares for error and blocks as reported in Marini (2003). The treatment structure for the apple experiment reported in Marini (2003) is shown in Table 1. It involves a combination of nesting and crossing of factors (Nelder and McCullagh, 1989). The simulated data is shown in Table 2. Due to rounding errors, the sums-of-squares in ANOVA tables are not exactly the same as in Marini (2003).

Firstly, the treatments fall into two groups: the control and the six treatments. This grouping may be represented by a dichotomous factor contr_vs_trt defined as in Table 2. Secondly, nested within the six treatments, there is a crossed two-way $3 \times 2$ treatment structure involving the factors formulation (form) 
Table 1. Treatment structure and simple means of apple experiment (Marini, 2003) and definition of factor contr_vs_trt.

\begin{tabular}{lccc}
\hline $\begin{array}{l}\text { Formulation } \\
\text { (form) }\end{array}$ & $\begin{array}{c}\text { Conc } \\
\left(\mathrm{mg} \cdot \mathrm{L}^{-1}\right)\end{array}$ & contr_vs_trt & $\begin{array}{c}\text { Fruit/100 } \\
\text { clusters }\end{array}$ \\
\hline Control & 0 & control & 35.7 \\
ABG-35 & 10 & trt & 50.1 \\
ABG-35 & 20 & trt & 66.9 \\
ABG-63 & 10 & trt & 55.2 \\
ABG-63 & 20 & trt & 80.3 \\
ABG-68 & 10 & trt & 62.3 \\
ABG-68 & 20 & trt & 58.9 \\
LSD $(5 \%)$ & & & 19.2 \\
\hline
\end{tabular}

Table 2. Simulated fruit set data based on apple experiment reported in Marini (2003). The simulation was designed to reproduce treatment means as well as the error and block sum of squares in Marini (2003).

\begin{tabular}{|c|c|c|c|c|c|c|c|}
\hline \multirow[b]{3}{*}{ Block } & \multicolumn{7}{|c|}{ Formulation/concn } \\
\hline & \multirow{2}{*}{$\begin{array}{c}\text { Control } \\
0\end{array}$} & \multicolumn{2}{|c|}{ ABG-35 } & \multicolumn{2}{|c|}{ ABG-63 } & \multicolumn{2}{|c|}{ ABG-68 } \\
\hline & & 10 & 20 & 10 & 20 & 10 & 20 \\
\hline 1 & 37.706 & 37.905 & 37.714 & 34.247 & 63.547 & 63.930 & 51.847 \\
\hline 2 & 19.970 & 32.049 & 53.435 & 10.901 & 81.702 & 64.321 & 64.519 \\
\hline 3 & 35.283 & 45.106 & 71.559 & 49.792 & 51.048 & 42.792 & 31.316 \\
\hline 4 & 14.197 & 35.652 & 34.345 & 71.225 & 55.520 & 58.537 & 57.421 \\
\hline 5 & 32.535 & 35.514 & 62.695 & 58.652 & 72.707 & 19.885 & 44.908 \\
\hline 6 & 74.509 & 114.374 & 141.651 & 106.382 & 157.276 & 124.336 & 103.390 \\
\hline
\end{tabular}

and concentration (conc). Using the syntax described in Piepho et al. (2003), which is essentially that given in McCullagh and Nelder (1989) and implemented in the statistical package GenStat, the two-way structure can be represented as

form $\times$ conc .

This resolves as

form + conc + form•conc ,

which is in a form directly usable in linear model packages. Some packages even allow specifying the model explicitly as form $\times$ conc (GenStat represents this as form*conc; in SAS the appropriate specification is form|conc). The nesting of the two-way structure within the grouping of control versus treatments (contr_vs_trt) can be expressed as

contr_vs_trt/(form $\times$ conc) ,

which expands as

contr_vs_trt + contr_vs_trt•form +

contr_vs_trt•conc + contr_vs_trt•form•conc.

Adding a block effect (block) this translates directly into the following statements in SAS, where the response variable is fruit set (set):

\section{PROC GLM:}

CLASS block contr_vs_trt form conc;

MODEL set $=$ block contr_vs_trt

contr_vs_trt*form contr_vs_trt*conc contr_vs_trt*form*conc;

RUN.

By comparison, the corresponding statements in GenStat are somewhat more compact and intuitive, because the model syntax allows direct specification of the nested treatment structure:

block block

treatment contr_vs_trt/(form*conc) anova [fprob=yes] set

The resulting ANOVA is shown in Table 3. Type I and Type III SS of SAS coincide here because the number of replications is the same for each of the seven treatments. There are highly significant differences between control and the mean of treatments. Interactions of the formulation $\times$ concentration treatment structure are not significant, so we consider the main effects. Only the main effects for concentration are significant, so it is appropriate to compare marginal means for that factor (Table 4). It is seen that the fruit set increases with concentration. Note that a comparison of simple formulation $\times$ concentration means would not be useful as the interaction is not significant. In addition, we compare the marginal means for the control and the group of six treatments (Table 4). The mean of the six treatments is markedly larger than the control. In summary, the higher concentration is to be preferred, regardless of the formulation used, and it is effective compared to the control.

The analysis reported here differs from those given by Marini (2003). In that paper, hypotheses are mostly tested by specifying single degree of freedom contrasts. In the present analysis, a full ANOVA is produced with all relevant sources of variation using a single model, so there is no need to specify contrasts, and the analysis is particularly simple to implement and straightforward to interpret. Instead of specifying contrasts, all relevant comparisons suggested by the ANOVA can be obtained from the least square means. It should also be noted that marginal means cannot be computed with any of the models used in Marini (2003), while the problem does not occur with the present approach.

Incidentally, fitting the model form $\times$ conc, ignoring the nesting within ctr_vs_trt, happens to produce the same ANOVA for the effects form, conc, and form-conc, as that reported in Table 3, if Type III SS are considered [also see Table 4 in Marini (2003)]. Of course, a test for contr_vs_trt is not produced with this model, and one cannot compute simple means or marginal means. Also, the Type I SS for form, conc, and form •conc are not the same as with Type III SS. With Type III SS, the test for form is adjusted for conc, as fitting conc takes out the control when coding factors as in Table 2. Similarly, the test for conc is adjusted for form, because fitting of form takes out the control. As a result, the Type III ANOVA for the model form $\times$ conc turns out to be that for the $3 \times 2$ factorial subdesign. But this coincidence will not be immediately obvious to the average user of Type III SS, nor does adherence to Type III SS provide a general strategy to deal with standards in complex factorial designs. It seems much more stringent and transparent to use the nested model contr_vs_trt/(form $\times$ conc), as this properly reflects all nesting and crossing features of the design.

Concentration is a quantitative factor, so one may also consider a regression (Marini, 2003). When fitting a regression model, conc needs to be declared as a quantitative variable (by removing it from the CLASS statement in SAS). Animportant aspect in a regression analysis is that the control now takes on a different role: it is the 0 concentration for the regression of each formulation. Due to the different role of the control, the model no longer contains the factor contr_vs_trt. Also, for any regression model it must be assumed that the curves of the three formulations have the same intercept. This is illustrated in Fig. 1. For this reason, the model does not have a formulation main effect(form). This main effect would imply a formulation-specific intercept, which is inappropriate, and it would effectively remove the control from the regression, as in the analysis provided by Marini (2003). The model

Table 3. Analysis of variance for apple experiment.

\begin{tabular}{llrrrr}
\hline Source & Term in SAS model statement & df & SS $^{z}$ & MS & F \\
\hline Block & block & 5 & 29172 & 5834 & 22.01 \\
Control vs. treatments & contr_vs_trt & 1 & 3634 & 3634 & 13.71 \\
Formulation & contr_vs_trt*form & 2 & 564 & 282 & 1.0001 \\
Concentration & contr_vs_trt*conc & 1 & 1482 & 1482 & 0.0009 \\
Formulation $\times$ concentration & contr_vs_trt*form*conc & 2 & 1289 & 645 & 0.3576 \\
Error & & 30 & 7956 & 265 & 0.0247 \\
\hline
\end{tabular}

${ }^{2}$ Type I and Type III SS in SAS are the same, when conc is a qualitative factor, because each of the seven treatments has the same number of replications. With unbalanced data the SS would differ. Type I SS would then be preferable due to better power (Nelder, 1994), but two different fitting sequences are required to obtain all relevant $\mathrm{F}$ tests (also see Example 2 for a discussion of Type I vs. Type III SS). 
Table 4. Fruit set marginal means for concentration and for control vs. treatments in apple experiment.

\begin{tabular}{lcc}
\hline Term & Level & Least square mean \\
\hline Concentration (contr_vs_trt•conc) & 0 & $35.7 \mathrm{a}$ \\
& 10 & $55.9 \mathrm{~b}$ \\
Control vs. treatments (contr_vs_trt) & 20 & $68.7 \mathrm{c}$ \\
& Control & $35.7 \mathrm{a}$ \\
\hline
\end{tabular}

${ }^{2}$ Means for a term that are followed by a common letter are not significantly different according to a $t$ test.

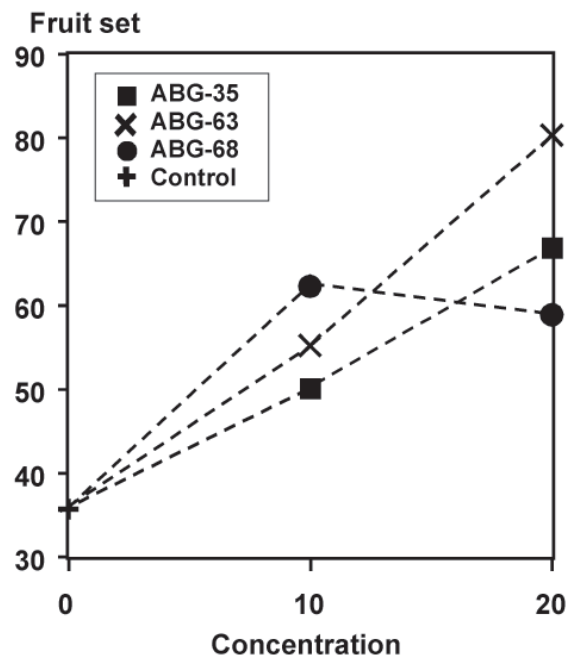

Fig. 1. Plot of treatment means for apple data as depending on gibberellin concentration and formulation.

suggested here does have formulation-specific regression effects. Each formulation has three concentrations, if the control is included, so a quadratic polynomial can be fitted. The model has the form (conc + conceconc)/form, which resolves as conc + conceconc + conceform + conceconc•form.

The crossed terms of conc and conc $\bullet$ conc with form provide tests for lack of homogeneity of the formulation-specific linear and quadratic regression coefficients. The only significant term in the sequential ANOVA (not shown) is the linear term (conc), so there is no heterogeneity among regression coefficients or indication of nonlinearity. Thus we could reduce the model and fit a single straight line to fully describe the results [The fitted regression line is: set $=$ $38.45+1.56 *$ conc] .

To illustrate the multiple comparison among regression coefficients, we also fit the model block + conc $\bullet$ form, despite the lack of heterogeneity among regressions. The regression coefficient estimates for the three formulations, along with multiple comparison results, are reported in Table 5. The regression coefficients are obtained from the solution vector for the model block + conc $\bullet$ form. Note that the factor form has four levels (Table 2): one for each of the three formulations and one for the control. Nevertheless, the term conc-form corresponds to only three estimable regression coefficients for the three gibberellin formulations. When fitting the model with PROC GLM, however, an additional regression coefficient is produced for the control (not shown). For this coefficient, an estimate of 0 is returned, and no associated standard error is printed, because no separate regression coefficient is estimable for the con-

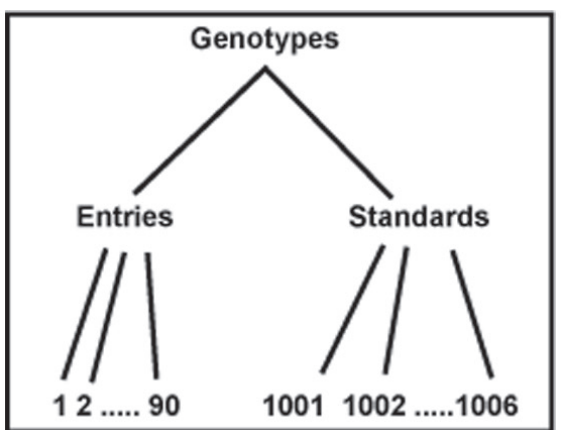

Fig 2. Hierarchical treatment structure of maize experiment.

trol. The regression coefficient for the control should be ignored in interpreting the results. None of the slopes for the three formulations are significantly different (Table 5), and this agrees with the nonsignificant heterogeneity among slopes found by an F test. Comparison among slopes required multiple ESTIMATE statements. The following SAS code was used to obtain this analysis:

PROC GLM;

CLASS block form;

MODEL set=block form* conc/solution; ESTIMATE 'slope 35-63' form*conc 1 -1 0 0;

ESTIMATE 'slope 35-68' form*conc 10 -10 ;

ESTIMATE 'slope 63-68' form*conc 01 $-10$

RUN.

\section{Example 2}

The treatment structure is hierarchical, as visualized in Fig. 2. The treatments (genotypes; geno) fall into two groups: entries and standards. The standards are coded as geno $=1001, \ldots$, 1006 , while entries are coded as geno $=1, \ldots$, 90. The dichotomy of standards versus entries may be defined by a factor stand_vs_entry
Table 5. Multiple comparison among regression slopes for apple experiment.

\begin{tabular}{lc}
\hline Formulation & Slope $^{\mathrm{z}}$ \\
\hline 35 & 1.37 \\
63 & 2.01 \\
68 & 1.30 \\
SED & 0.44
\end{tabular}

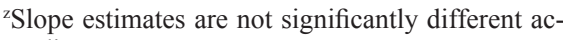
cording to a $t$ test.

${ }^{\mathrm{y}} \mathrm{SED}=$ standard error of a difference.

with a different level for both groups (Table 6). To compare standards in an ANOVA, we define a factor standards, which has a separate level for each standard and a common level for all entries (0). If this factor is nested within stand_vs_entry, the ANOVA will yield a test of the null hypothesis that all standards have the same yield. In an analogous fashion, we define a factor entries for the entries. The treatment structure can then be written as

stand_vs_entry/(standards + entries),

which expands as

stand_vs_entry +

stand_vs_entry•standards +

stand_vs_entry•entries

The randomization structure can be represented as rep/block, where rep codes complete replications, while blocks are coded by block (Piepho et al., 2003). A full ANOVA, which takes all effects as fixed, is shown in Table 7. The assumption of fixed block effects results in an intra-block analysis that does not recover inter-block information. Two different orders of fitting effects are needed when using Type I SS. The correct test for standards is obtained by fitting standards last in the sequence of effects. Conversely, entries need to be fitted last to obtain the correct test for entries. Type III SS yield the correct test for both effects. The tests for stand_vs_entry also differ between Type I and Type III SS. In the present case, Type III $\mathrm{SS}$ give equal weight to each genotype within a group, while Type I SS accounts for unequal sample sizes. In case there are real differences among standards or among entries, this causes the hypothesis being tested to depend on the design (Searle, 1987), which is not desirable. Type III SS may be preferable here, because the F test for stand_vs_entry is equivalent to a contrast test among the least square means for standards vs. entries. Alternatively, one can argue that a comparison of the mean of standards versus the mean of entries will be meaningful only if there are no differences among entries and among

Table 6. Treatment structure and definition of treatment factors for maize experiment.

\begin{tabular}{|c|c|c|c|c|}
\hline Genotype (geno) & Group & stand_vs_entry & standards & entries \\
\hline 1 & Entry & 1 & 0 & 1 \\
\hline 2 & Entry & 1 & 0 & 2 \\
\hline 3 & Entry & 1 & 0 & 3 \\
\hline • & - & - & $\bullet$ & - \\
\hline • & • & • & $\bullet$ & • \\
\hline - & - & - & $\bullet$ & - \\
\hline 89 & Entry & 1 & 0 & 89 \\
\hline 90 & Entry & 1 & 0 & 90 \\
\hline 1001 & Standard & 2 & 1001 & 0 \\
\hline 1002 & Standard & 2 & 1002 & 0 \\
\hline 1003 & Standard & 2 & 1003 & 0 \\
\hline 1004 & Standard & 2 & 1004 & 0 \\
\hline 1005 & Standard & 2 & 1005 & 0 \\
\hline 1006 & Standard & 2 & 1006 & 0 \\
\hline
\end{tabular}


standards. In this case, Type I and Type III SS test the same hypothesis, and Type I SS will have the advantage of better power (Nelder, 1994).

With regard to the comparison among standards and among entries, note that the treatment design may be viewed as being composed of two nonoverlapping subdesigns. This nonoverlap is the prerequisite for a single joint analysis of both subdesigns by a single model. By contrast, in Example 3 the two subdesigns of interest are overlapping, so two separate analyses are necessary.

Table 7. Analysis of variance for maize experiment.

\begin{tabular}{|c|c|c|c|c|c|c|}
\hline Source & Term in SAS model statement & $\mathrm{df}$ & Type & MS & $\mathrm{F}$ & $P>\mathrm{F}$ \\
\hline & & & Type III SS & & & \\
\hline Replications & rep & 1 & 37062 & 37062 & 30.68 & $<0.0001$ \\
\hline Block, nested in replications & rep*block & 18 & 48895 & 2716 & 2.25 & 0.0071 \\
\hline Standards vs. entries & stand_vs_trt & 1 & 103451 & 103451 & 85.65 & $<0.0001$ \\
\hline Standards & stand_vs_trt*standards & 5 & 56352 & 11270 & 9.33 & $<0.0001$ \\
\hline Entries & stand_vs_trt*entries & 89 & 524102 & 5889 & 4.88 & $<0.0001$ \\
\hline \multirow[t]{2}{*}{ Error } & & 83 & 100251 & 1208 & & \\
\hline & & & Type I SS & & & \\
\hline Replications & rep & 1 & 37062 & 37062 & 30.68 & $<0.0001$ \\
\hline Block, nested in replications & rep*block & 18 & 48895 & 2716 & 2.25 & 0.0071 \\
\hline Standards vs. entries & stand_vs_trt & 1 & 70910 & 70910 & 58.71 & $<0.0001$ \\
\hline Standards, fitted before entries & stand_vs_trt*standards & 5 & 62230 & 12446 & 10.30 & $<0.0001$ \\
\hline Entries, fitted after standards & stand_vs_trt*entries & 89 & 524102 & 5889 & 4.88 & $<0.0001$ \\
\hline \multirow[t]{2}{*}{ Error } & & 83 & 100251 & 1208 & & \\
\hline & & & Type I SS & & & \\
\hline Replications & rep & 1 & 37062 & 37062 & 30.68 & $<0.0001$ \\
\hline Block, nested in replications & rep*block & 18 & 48895 & 2716 & 2.25 & 0.0071 \\
\hline Standards vs. entries & stand_vs_trt & 1 & 70910 & 70910 & 58.71 & $<0.0001$ \\
\hline Entries, fitted before standards & stand_vs_trt*entries & 89 & 529980 & 5955 & 4.93 & $<0.0001$ \\
\hline Standards, fitted after entries & stand_vs_trt*standards & 5 & 56352 & 11270 & 9.33 & $<0.0001$ \\
\hline Error & & 83 & 100251 & 1208 & & \\
\hline
\end{tabular}

Table 8. Treatment structure for carrot experiment. Factor variable names are boldfaced.

\begin{tabular}{|c|c|c|c|c|c|c|c|}
\hline \multirow{2}{*}{$\begin{array}{l}\text { Nitrogen } \\
\text { (n) }\end{array}$} & \multirow{2}{*}{$\begin{array}{c}\text { Light (\%) } \\
\text { (light) }\end{array}$} & \multirow{2}{*}{$\begin{array}{c}\text { Substrate } \\
\text { (sub) }\end{array}$} & \multirow{2}{*}{$\begin{array}{c}\text { Horn silica } \\
\text { (hs) }\end{array}$} & \multirow{2}{*}{$\begin{array}{c}\text { Horn manure } \\
(\mathbf{h m})\end{array}$} & \multirow{2}{*}{$\begin{array}{c}\text { Treatment } \\
\text { (trt) }\end{array}$} & \multicolumn{2}{|c|}{ Selector variables } \\
\hline & & & & & & w1 & w2 \\
\hline \multirow[t]{16}{*}{ With (1) } & $100(1)$ & Loamy soil (1) & Without (1) & Without (1) & 1 & 0 & 0 \\
\hline & & & & With (2) & 2 & 0 & 0 \\
\hline & & & With (2) & Without (1) & 3 & 0 & 0 \\
\hline & & & & With (2) & 4 & 0 & 0 \\
\hline & & Sand (2) & Without (1) & Without (1) & 5 & 0 & 5 \\
\hline & & & & With (2) & 6 & 0 & 6 \\
\hline & & & With (2) & Without (1) & 7 & 0 & 7 \\
\hline & & & & With (2) & 8 & 0 & 8 \\
\hline & $75(2)$ & Loamy soil (1) & Without (1) & Without (1) & 9 & 0 & 9 \\
\hline & & & & With (2) & 10 & 0 & 10 \\
\hline & & & With (2) & Without (1) & 11 & 0 & 11 \\
\hline & & & & With (2) & 12 & 0 & 12 \\
\hline & & Sand (2) & Without (1) & Without (1) & 13 & 0 & 13 \\
\hline & & & & With (2) & 14 & 0 & 14 \\
\hline & & & With (2) & Without (1) & 15 & 0 & 15 \\
\hline & & & & With (2) & 16 & 0 & 16 \\
\hline \multirow[t]{4}{*}{ Without (2) } & $100(1)$ & Loamy soil (1) & Without (1) & Without (1) & 17 & 17 & 0 \\
\hline & & & & With(2) & 18 & 18 & 0 \\
\hline & & & With (2) & Without (1) & 19 & 19 & 0 \\
\hline & & & & With (2) & 20 & 20 & 0 \\
\hline
\end{tabular}

Table 9. Four-way ANOVA (light $\times$ substrate $\times$ horn silica $\times$ horn manure) for carrot experiment.

\begin{tabular}{|c|c|c|c|c|c|c|}
\hline Source & Term in SAS model statement & $\mathrm{df}$ & $\mathrm{SS}^{\mathrm{z}}$ & MS & $\mathrm{F}$ & $P>\mathrm{F}$ \\
\hline Take out treatments without nitrogen fertilizer & $\mathbf{w 1}$ & 4 & 16703.04 & 4175.76 & 91.63 & $<0.0001$ \\
\hline Light & light*w1 & 1 & 3607.50 & 3607.50 & 79.16 & $<0.0001$ \\
\hline Substrate & sub*w1 & 1 & 123.49 & 123.49 & 2.71 & 0.1050 \\
\hline Light $\times$ substrate & light*sub*w1 & 1 & 240.64 & 240.64 & 5.28 & 0.0251 \\
\hline Horn silica & hs*w1 & 1 & 77.22 & 77.22 & 1.69 & 0.1980 \\
\hline Light $\times$ horn silica & light*hs*w1 & 1 & 60.26 & 60.26 & 1.32 & 0.2548 \\
\hline Substrate $\times$ horn silica & sub*hs*w1 & 1 & 22.21 & 22.21 & 0.49 & 0.4878 \\
\hline Light $\times$ substrate $\times$ horn silica & light*sub*hs*w1 & 1 & 11.99 & 11.99 & 0.26 & 0.6099 \\
\hline Horn manure & hm*w1 & 1 & 26.39 & 26.39 & 0.58 & 0.4496 \\
\hline Horn manure $\times$ light & light*hm*w1 & 1 & 25.13 & 25.13 & 0.55 & 0.4607 \\
\hline Horn manure $\times$ substrate & sub*hm*w1 & 1 & 8.05 & 8.05 & 0.18 & 0.6757 \\
\hline Horn manure $\times$ light $\times$ substrate & light*sub*hm*w1 & 1 & 0.26 & 0.26 & 0.01 & 0.9397 \\
\hline Horn manure $\times$ horn silica & hs*hm*w1 & 1 & 1.59 & 1.59 & 0.03 & 0.8523 \\
\hline Horn manure $\times$ light $\times$ horn silica & light*hs*hm*w1 & 1 & 0.02 & 0.02 & 0.00 & 0.9838 \\
\hline Horn manure $\times$ substrate $\times$ horn silica & sub*hs*hm*w1 & 1 & 78.99 & 78.99 & 1.73 & 0.1930 \\
\hline Horn manure $\times$ light $\times$ substrate $\times$ horn silica & light*sub*hs*hm*w1 & 1 & 29.03 & 29.03 & 0.64 & 0.4280 \\
\hline$\underline{\text { Error }}$ & & 60 & 2734.27 & 45.57 & & \\
\hline
\end{tabular}

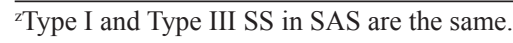


Table 10. Single root mass means $[\mathrm{g}]$ for substrate $\times$ light in carrot experiment.

\begin{tabular}{lcc}
\hline & \multicolumn{2}{c}{ Light $^{\mathrm{z}}(\%)$} \\
\cline { 2 - 3 } Substrate $^{\mathrm{y}}$ & 100 & 75 \\
\hline Loamy soil & $89.46 \mathrm{aA}$ & $70.56 \mathrm{aB}$ \\
Sand & $82.80 \mathrm{bA}$ & $71.66 \mathrm{aB}$ \\
SED $^{\mathrm{x}}$ & 2.39 & \\
\hline
\end{tabular}

${ }^{\mathrm{z}}$ Means in a column followed by the same lowercase letter are not significantly different according to a $t$ test.

${ }^{y}$ Means in a row followed by the same upper-case letter are not significantly different.

${ }^{\mathrm{x}} \mathrm{SED}=$ standard error of a difference.

\section{Example 3}

The design for the carrot experiment was analysed as a completely randomized design with four replications. The light factor could not be randomised and thus may be confounded with other environmental factors. The carrot data give rise to two overlapping subdesigns: the $2^{4}$ factorial light $\times$ substrate $\times$ horn silica $\times$ horn manure for the subset of treatments fertilized with nitrogen and the $2^{3}$ factorial nitrogen $x$ horn silica $x$ horn manure for the subset of all treatments with $100 \%$ light and loamy soil. For the $2^{4}$ factorial, a four-way ANOVA of light $\times$ substrate $\times$ horn silica $\times$ horn manure is required, ignoring all unfertilized treatments, while for the $2^{3}$ factorial a three-way ANOVA of nitrogen $\times$ horn silica $\times$ horn manure is required, ignoring treatments with $75 \%$ light or sandy soil. As can be seen from Table 8, the two designs are overlapping: they share the four treatments with fertilizer, 100\% light, and loamy soil (first four treatments in Table 8). Thus, two separate model fits are needed. The treatment factors are coded light, sub, n, $\mathbf{h s ,}$, and $\mathbf{h m}$ for light, substrate, nitrogen, horn silica, and horn manure, respectively. Arunning number (trt) is defined for uniquely identifying all 20 treatments. The analyses may be done by defining additional factors (Table 8).

Light $\times$ substrate $\times$ horn silica $\times$ horn manure ANOVA: In order to select the subset of treatments in the four-way classification, we define a new variable w1, which is equal to 0 for all treatments of interest and equal to trt for all treatments not receiving nitrogen. Note that the particular value assigned to w1 for each level is not important. The important property of w 1 is that all treatments of interest have the same level, whereas each treatment to be excluded has a different level. The variable w1 can then be used to block out the treatments without nitrogen from the four-way ANOVA. Nested within w1, we specify the four-way model. The treatment structure for the four-way ANOVA has the form

w1 $/($ light $\times$ sub $\times$ hs $\times$ hm)

The resulting ANOVA is given in Table 9. Note that Type I and Type III SS coincide in this case, because the four-way subdesign is orthogonal. The only significant effects are light and light'sub. Thus, we compute the least square means for light $\bullet$ sub and compare light regimes for levels of substrate and vice versa (Table 10). All pairwise comparisons are significant, except that between sand and loamy soil at $75 \%$ light. It emerges that light was the limiting factor under the shaded regime $(75 \%$ light), while with full light the better soil led to improved growth. This pattern nicely explains the light $\times$ substrate interaction. It should be stressed that it would be misleading to compare marginal means for light or substrate, because the interaction was significant. Clearly, a comparison of marginal means would miss a central feature of the data. The SAS statements are as follows:

\section{PROC GLM;}

\section{CLASS light sub hs hm w1;}

MODEL srmass $=\mathrm{w} 1$

w1*light

w1*sub

w1*light*sub

w1*hs

w1*hs*light

w1*hs*sub

w1*hs*light*sub

w1*hm

w1*hm*light

w1*hm*sub

w1*hm*light*sub

w1*hm*hs

w1*hm*hs*light

w1 1 hm*hs*sub

w1*hm*hs*light*sub/SS1;

LSMEANS w1 *light*sub/PDIFF; RUN.

The analysis could also be obtained with the new GLIMMIX procedure of SAS, using exactly the same statements as with GLM (dropping the SS1 option from the model statement). A very useful new feature of GLIMMIX is the SLICEDIFF option. For example, comparisons of w1 $\cdot$ light $\bullet$ sub means at the same level of substrate would be obtained by adding SLICEDIFF $=\mathbf{w} 1 *$ sub to the LSMEANS statement.

Nitrogen $\times$ horn silica $\times$ horn manure ANOVA: In analogy to the four-way ANOVA a new variable w2 is defined, which is equal to 0 for all treatments of interest (100\% light, loamy soil) and equal to trt otherwise. This variable takes out treatments with $75 \%$ light or sand from the three-way ANOVA. The three-way model is then nested within w2. The treatment structure for the three-way ANOVA has the form $\mathbf{w} \mathbf{2} /(\mathbf{n} \times \mathbf{h s} \times \mathbf{h m})$.

The resulting ANOVA is given in Table 11. Again Type I and Type III SS coincide, because the three-way subdesign is orthogonal. The only significant effect is nitrogen, so we compute the corresponding marginal means (Table 12). Fertilization more than doubles single root mass compared to the mean of fertilizer-deficient treatments (without fertilizer).

The ANOVAs for the two subdesigns revealed significant effects only for light, light $\bullet$ substrate, and nitrogen, while no significant main effects or interactions were found for horn manure or horn silica. Neither of these analyses used the full information on horn silica and manure, as far as main effects are concerned, because the focus was on the orthogonal subdesigns. In case of nonsignificant interaction of horn manure and silica with factors light, substrate and nitrogen, it is useful to perform an ANOVA for horn silica $\times$ horn manure, adjusting for significant effects in the light $\times$ substrate $\times$ nitrogen classification. In the present case, this amounts to fitting the model light•sub $+\mathbf{n}+\mathbf{h s} \times \mathbf{h m}$. The Type III SS yield nonsignificant $\mathrm{p}$-values for $\mathbf{h s}, \mathbf{h m}$, and hs $\bullet$ hm $(P=0.208, P=0.365$, and $P=0.901$, respectively). The means for factors $\mathbf{h s}$ and $\mathbf{h m}$ are quite different among the different analyses, i.e., for the $2^{4}$ and $2^{3}$ factorials and the present analysis that used all information from both subdesigns. Table 13 bears this out for the horn silica means. The reason for this difference is that treatments included differ in the levels of the other factors. By contrast, differences among means are of the same order of magnitude, if related to their standard errors.

So far, all treatment effects have been taken as fixed. If a factor has many levels, which may be regarded as a random sample from some parent distribution, it may be preferable to analyse the associated effects as random (Searle et al., 1992; Piepho, 1998). In plant breeding trials one will be interested in estimating random effects, which is done by best linear unbiased prediction (BLUP; Searle et al., 1992; Panter and Allen, 1995). For the maize data, one may want to take entries as random, while standards are taken as fixed. The rationale for this approach is that standard varieties are expected to be different from the population of entries and it is not reasonable to regard them as a random

Table 11. Three-way ANOVA (nitrogen $\times$ horn silica $\times$ horn manure) for carrot experiment.

\begin{tabular}{|c|c|c|c|c|c|c|}
\hline Source & Term in SAS model statement & df & $\mathrm{SS}^{\mathrm{z}}$ & MS & $\mathrm{F}$ & $P>\mathrm{F}$ \\
\hline Take out treatments with $75 \%$ light or sand & w2 & 12 & 3313.83 & 276.15 & 6.06 & $<0.0001$ \\
\hline Horn silica & hs*w2 & 1 & 2.05 & 2.05 & 0.04 & 0.8327 \\
\hline Horn manure & $\mathrm{hm} * \mathrm{w} 2$ & 1 & 10.93 & 10.93 & 0.24 & 0.6261 \\
\hline Horn silica $\times$ horn manure & hs*hm*w2 & 1 & 26.10 & 26.10 & 0.57 & 0.4521 \\
\hline Nitrogen & $\mathrm{n} * \mathrm{w} 2$ & 1 & 17629.73 & 17629.73 & 386.86 & $<0.0001$ \\
\hline Nitrogen $\times$ horn silica & $\mathrm{n} * \mathrm{hs} * \mathrm{w} 2$ & 1 & 13.13 & 13.13 & 0.29 & 0.5934 \\
\hline Nitrogen $\times$ horn manure & $\mathrm{n} * \mathrm{hm} * \mathrm{w} 2$ & 1 & 2.48 & 2.48 & 0.05 & 0.8165 \\
\hline Nitrogen $\times$ horn silica $\times$ horn manure & $\mathrm{n} * \mathrm{hk} * \mathrm{hm} * \mathrm{w} 2$ & 1 & 17.55 & 17.55 & 0.39 & 0.5372 \\
\hline Error & & 60 & 2734.27 & 45.57 & & \\
\hline
\end{tabular}

${ }^{2}$ Type I and Type III SS in SAS are the same. 
Table 12. Marginal single root mass means [g] for fertilizer in carrot experiment.

\begin{tabular}{lc}
\hline Fertilizer & Mean $^{\mathrm{z}}$ \\
\hline With & $89.46 \mathrm{a}$ \\
Without & $42.51 \mathrm{~b}$ \\
SED $^{\mathrm{y}}$ & 4.77 \\
\hline
\end{tabular}

${ }^{2}$ Means followed by a common letter are not significantly different according to a $t$ test.

${ }^{y} \mathrm{SED}=$ standard error of a difference.

sample from that population (Piepho and Pillen, 2004). Furthermore, BLUP is designed to maximize the probability of correct ranking among effect estimates (Searle et al., 1992), and it is reasonable to do so only for those entries among which selections are to be made, thus excluding standards. Model specification is similar to the case when all treatment effects are fixed. Essentially, the entry effects are moved from the fixed to the random part. Thus, the effect stand_vs_entry•entries could be declared as a random effect. The resulting model is not quite correct, however, because the effect will have a separate level for all entries plus an additional level for standards. Specifically, standards would have a genetic variance component. Clearly, this variance structure would be misspecified, as we want standards to be associated with no random genetic effect, while all entries have a separate random effect. This may be achieved by defining a dummy variable $\mathbf{z}$ so that $\mathbf{z}=\mathbf{0}$ for standards and $\mathbf{z}=\mathbf{1}$ for entries. The variable $z$ is defined as a quantitative rather than a qualitative factor. In SAS this requires omitting $\mathbf{z}$ from the list of CLASS factors. The random entry effect may then be specified as z•entries. This effect induces a genetic variance of zero for all standards and a common genetic variance for all entries. Care must be taken so that $\mathbf{z}$ is not automatically mean-centered by the statistical package. The statements for the MIXED procedure are as follows, using the Kenward-Roger method to adjust the Wald F statistic and degrees of freedom, and taking blocks as random for recovery of inter-block information:

\section{PROC MIXED;}

CLASS rep block stand_vs_entry standards entries;

MODEL yield=rep stand_vs_entry stand_vs_entry*standards/DDFM=KR; RAND̄OM rep*block $\mathrm{z}^{*}$ entries; RUN;

It should be stressed that the model would not be strictly correct if $\mathrm{z}$ were declared as a qualitative factor (CLASS variable), because this would also induce a random genetic effect for the group of standards, thus inflating the variance associated with observations in that group. This can best be seen by the resulting change in the Type III Wald F test for the factor stand vs entry. With the assumption of random entries, the null hypothesis tested here is that the mean of the standards equals the expected value of the parent population from which entries have been sample. Note that this is not quite the same as under a model with fixed entries, where the null hypothesis states equality of the mean of standards and the mean of tested entries. When $\mathbf{z}$ is a continuous variable, the
Wald $\mathrm{F}$ value for this test is 67.51 , which is highly significant $(P<0.0001)$, while when $\mathbf{z}$ declared as a qualitative factor (CLASS variable), the Wald $\mathrm{F}$ value drops to 3.33 and is not significant $(P=0.0729)$. Essentially this occurs because the model mis-specification leads to an inflated error term for the standards. Interestingly, the test forstand_vs_entry is the only part of the analysis that is affected by declaring $\mathrm{z}$ as a qualitative factor. Everything else is identical between both analyses, including all parameter estimates, the log-likelihood, and all BLUPs of random effects. The reason is that standards provide no degrees of freedom for the genetic variance in either case as all genetic variation is accounted for by the fixed effects structure, while the models differ in the variance structure implied for the standards, thus affecting the Wald F value for stand_vs_entry.

With Examples 2 and 3, we have not presented the corresponding GenStat code. Comparative examples of SAS and GenStat code are given, however, by Williams et al. (2002). The SAS code for all three examples is available from the first author upon request.

\section{Concluding Remarks}

Treatment structures deviating from simple textbook examples are often quite useful in answering complex research questions. It is not always obvious, however, in which way such designs should be analysed. A common recommendation is to fit a one-way model and define linear contrasts for hypotheses of interest (Perry, 1986; Marini, 2003; Frömke and Bretz, 2004), and this is indeed generally a viable option. In fact, use of contrasts is often the most useful approach, e.g., when there is a complex design for a set of standards with logical order (Hothorn and Bleiholder, 2006) or when heterosis effects in breeding trials or mixture effects in inter-cropping experiments are to be estimated. In many cases, however, this approach is somewhat tedious. In Example 2 , one would have to define a contrast with 96 coefficients to compare the mean of standards to the mean of entries. It often turns out that an essentially equivalent analysis can be obtained from an ANOVA, followed by mean comparisons, if a suitable parameterization of the linear model is used (Williams et al., 2002). This paper has discussed three such cases, and in all of them the key feature of the model used was a nested treatment structure. Also, with carefully defined model effects, it was straightforward to derive all least square means of interest.

It is worth mentioning that the factor sepa-

Table 13. Means and differences for horn silica under different treatment models.

\begin{tabular}{lccc}
\hline Treatment model & Level of horn silica & Estimate & SE \\
\hline $\mathbf{w 1} /($ light $\times$ sub $\times \mathbf{h s} \times \mathbf{h m})$ & Without & 79.7 & 1.19 \\
& With & 77.5 & 1.19 \\
$\mathbf{w} 2 /(\mathbf{n} \times \mathbf{h s} \times \mathbf{h m})$ & Difference & 2.2 & 1.69 \\
& Without & 65.7 & 1.69 \\
& With & 66.2 & 1.69 \\
light* $\mathbf{s u b}+\mathbf{n}+\mathbf{h s} \times \mathbf{h m}$ & Difference & -0.5 & 2.39 \\
& Without & 56.1 & 1.35 \\
& With & 54.2 & 1.35 \\
& Difference & 1.9 & 1.44 \\
\hline
\end{tabular}

rating treatments of interest from controls or factors pertaining to other subdesigns need not be at the top of the factorial hierarchy, i.e., it need not be crossed with all other treatment effects a field experiment evaluating three different types of fertilizer(fert) plus a control treatment. If $\mathbf{w}$ is a dichotomous variable separating the control from fertilizer treatments, this yields the treatment structure w/fert. Now assume that on each plot some soil property is measured at three different depths (depth). This factor needs to be crossed with $\mathbf{w} /$ fert, resulting in the model (w/fert) $\times$ depth, which expands to $\mathbf{w}$

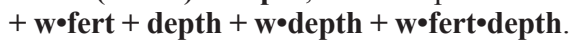
It is seen that the model has a main effect for depth that is not crossed with $\mathbf{w}$.

It should be emphasized that the approach advocated in this paper is strongest when the treatment structure has been carefully designed. At the design stage, one should also think about how exactly the data are going to be analysed and how the model is to be parameterised. This will often involve an iterative process, in which the treatment structure is successively fine-tuned to address all relevant research questions. Developing the model in parallel can help clarifying the research objectives and finding a useful treatment design. Conversely, difficulty in specifying an appropriate model can indicate that the treatment design is not entirely appropriate for the purpose of the experiment. As our examples have illustrated, specification of a model requires that additional factors be suitably defined by the user. This may not always a straightforward exercise, and if in doubt a statistician should be consulted.

Our examples mainly required the use of fixed effects models with normal errors. The suggested approach to modelling the treatment structure is applicable to any linear model, including linear mixed models (LMM), which allow great flexibility in accounting for multiple random sources of variation, as occur, e.g., with split-plot designs, and generalized linear mixed models(GLMMs), which can also handle nonnormal errors. LMMs and GLMMs may be fitted, e.g., with the MIXED and GLIMMIX procedures of the SAS System. These procedures, especially GLIMMIX, provide improved capabilities for comparisons among adjusted means.

In the example we touched upon the issue of sequential sums-of-squares (Type I SS in SAS) versus other types such as Type III SS. All our comments in this regard apply equally to Type I and Type III hypotheses tested in a generalized linear and/or mixed model, where sums-of-squares are not or cannot be computed. appearing in a model. For example, consider 
More often than not there is a natural order for the terms in the analysis. For example, one will generally want to fit blocks before treatments. Similarly, in Example 2 it seems natural to fit standards before entries, because the main interest is usually in the entries. Power considerations also lead to a general preference for Type I SS (Nelder, 1994). There are, however, situations, where Type III SS may be useful, e.g. in multiple regression. The test for control vs. treatments in Example 2 turned out to be more meaningful with Type III SS if significant differences are assumed between standards or between entries. The same test can be obtained, however, by a $t$ test comparing marginal means of standards and entries. Thus, it does not seem possible to give very simple rules here. Nevertheless, it is our experience that most experiments can be very efficiently analysed with Type I SS (Williams et al., 2002). When the design is orthogonal in some sense, both types typically yield identical results.

The use of multiple comparisons in factorial experiments is often discouraged (Perry, 1986), and some scientific journals as well as official guidelines for evaluation trials (e.g., Anonymous, 1998) explicitly make recommendations to this effect. The recommendation seems to be mostly a reaction to the common but unfortunate practice of analysing a twofactorial experiment by a one-way treatment model, using multiple pairwise comparisons among all treatments. This practice is indeed to be discouraged as it ignores the inherent treatment structure. It is not, however, the multiple comparison as such that is inappropriate, it is the type of multiple comparison performed that is problematic (Hothorn and Bleiholder, 2006). Clearly, if the treatment design has a two-way structure, the data should be analysed accord- ingly. If the interaction is not significant, it is useful to do a multiple comparison of marginal means for both factors, while with significant interaction, multiple comparisons are needed among levels of one factor, fixing the level of the other factor. Another argument frequently put forward against the use of multiple comparison procedures is that often at least one factor of interest is quantitative, e.g., different levels of fertilizer. In this case a regression is often more meaningful than a multiple comparison of means. One may, however, wish to perform multiple comparisons among regression coefficients, and Example 1 gives an illustration. Thus, presence of quantitative treatment factors in a design does not rule out the need for multiple comparisons. If one is prepared to look at multiple comparisons, a crucial and long-debated issue is the appropriate error rate, the most common choices being the comparison-wise and the experiment-wise Type I error rate (Frömke and Bretz, 2004; Saville, 2003). In examples, we have controlled only the comparison-wise error rate for simplicity. Regardless of continuing controversies revolving around the choice of error rate, we believe that multiple comparison procedures do have an important role to play in the analysis of factorial treatment structures. As was illustrated in this paper, a suitable definition of model terms may considerably facilitate the computation of treatment means and their pairwise comparison in complex factorial designs.

\section{Literature Cited}

Frömke, C. and F. Bretz. 2004. Simultaneous tests and confidence intervals for the evaluation of agricultural field trials. Agron. J. 96:1323-1330.

Hothorn, L.A. and H. Bleiholder. 2006 Statistical aspects of efficacy evaluation of plant protection products in field trials - A comment on the EPPO PP1/152(2) guideline (in press)

Littell, R.C., W.W. Stroup, and R. Freund. 2002. SAS for linear models. 4th ed. SAS Inst., Cary, N.C.

Marini, R.P. 2003. Approaches to analyzing experiments with factorial arrangements of treatments plus other treatments. HortScience 38:117-120.

McCullagh, P. and J.A. Nelder. 1989. Generalized linear models. 2nd ed. Chapman \& Hall, London.

Nelder, J.A. 1994. The statistics of linear models: back to basics. Stat. Computing 4:221-234.

OEPP/EPPO. 1998. OEPP/EPPO: Design and analysis of efficacy evaluation trials, EPPO Standard PP1/152(2). Bul. OEPP/EPPO 28:273-348.

Panter, D.M. and F.L. Allen. 1995. Using best linear unbiased predictions to enhance breeding for yield in soybean. 2. Selection of superior crosses from a limited number of yield trials. Crop Sci. 35:405-410.

Perry, J.N. 1986. Multiple-comparison procedures: a dissenting view. J. Econ. Entomol. 79:1149-1155.

Piepho, H.P. 1998. Empirical best linear unbiased prediction in cultivar trials using factor analytic variance-covariance structures. Theor. Appl. Genet. 97:195-201.

Piepho, H.P., A. Büchse, and K. Emrich. 2003. A hitchhiker's guide to the mixed model analysis of randomized experiments. J. Agron. Crop Sci. 189:310-322.

Piepho, H.P. and K. Pillen. 2004. Mixed modelling for QTL $\times$ environment interaction analysis. Euphytica 137:147-153.

Saville, D. 2003. Basic statistics and the inconsistency of multiple comparison procedures. J. Expt. Psychol. 57:167-175.

Searle, S.R. 1987. Linear models for unbalanced data. Wiley, New York.

Searle, S.R., G. Casella, and C.E. McCulloch. 1992. Variance components. Wiley, New York.

Williams, E.R., A.C. Matheson, and C.E. Harwood. 2002. Experimental design and analysis for tree improvement. 2nd ed. CSIRO Publ., Canberra. 